\title{
On the Pilot Carrier Placement in Multicarrier-Based Systems
}

\author{
Heidi Steendam, Senior Member, IEEE
}

\begin{abstract}
In traditional multicarrier systems, the pilot carriers used to estimate the channel are placed as uniform as possible over the bandwidth. However, with the raise of cognitive radio systems, where the multicarrier system is used by the secondary users, parts of the bandwidth are not available for transmission as primary users are active in these bands. Therefore, the multicarrier system must introduce guard bands in which the carriers may not be used. Hence, a problem might occur when placing the pilot carriers. In this paper, we investigate the effect of the positions of the pilot carriers on the MSE performance of channel estimation, and look for the pilot carrier placement that minimizes the MSE. We do not restrict our attention to multicarrier systems with a cyclic prefix, but we also consider other types of guard interval that are used for multicarrier transmission. It is known that an equidistantlyspaced distribution of the pilot carriers is in general not the optimal placement of the pilot carriers, as the corresponding MSE can become very high. In this paper, we use a heuristic algorithm to search for the best pilot placement, which is able to deliver a pilot carrier placement that outperforms the maximum distance distribution from [S. Song and A. C. Singer, "Pilot-Aided OFDM Channel Estimation in the Presence of the Guard Band," IEEE Trans. Commun., vol. 55, no. 8, pp. 1459-1465, Aug. 2008] in terms of the MSE, and results in an MSE that is close to the case where no guard bands are present. Based on the results of the algorithm, we can derive some simple rules of thumb to select the positions of the pilot carriers.
\end{abstract}

Index Terms-Carrier placement, channel estimation, guard interval, OFDM.

\section{INTRODUCTION}

M ULTICARRIER (MC) transmission [2] is one of the most popular transmission techniques ever, witness the many standards based on this technique, thanks to its robustness to channel dispersion. Since the advent of multicarrier transmission, much research has been devoted to channel estimation (see e.g. [3]). To be able to efficiently estimate time varying channels, typically pilot carriers are sparsely inserted in the time-frequency grid of the MC signal, and based on the observations of

Manuscript received January 11, 2013; revised September 04, 2013 and December 16, 2013; accepted February 09, 2014. Date of publication February 13, 2014; date of current version March 10, 2014. The associate editor coordinating the review of this manuscript and approving it for publication was Prof. Wolfgang Utschick. The author gratefully acknowledges the financial support from the Flemish Fund for Scientific Research (FWO). This research has been funded by the Interuniversity Attraction Poles Programme initiated by the Belgian Science Policy Office.

The author is with the DIGCOM research group, TELIN Department, Ghent University, 9000 Gent, Belgium (e-mail: Heidi.Steendam@telin.ugent.be).

Color versions of one or more of the figures in this paper are available online at http://ieeexplore.ieee.org.

Digital Object Identifier 10.1109/TSP.2014.2306179 these pilot carriers, the channel on the other (data) carriers is estimated. Commonly used pilot carrier insertion schemes are the block type and comb type pilot schemes [4]. Based on the pilot carrier observations, two approaches exist for estimating the overall channel. In the first approach, the underlying channel model is not taken into account, and interpolation is used to obtain the channel estimates for the intermediate data carriers. As the optimal 2D Wiener interpolation is hard to implement in practice, low complexity solutions as e.g. linear, quadratic or spline interpolation have been proposed [5]-[7]. Although these low complexity interpolation techniques are robust to the underlying channel model, the main drawback of these methods is the performance error floor they exhibit for the bit error rate (BER) at high signal-to-noise ratio (SNR).

In the second approach, the underlying channel model is assumed to be sparse in the sense that the channel can be modelled using a small number of channel taps, an assumption that turns out to be valid for most practical situations. Based on the pilot carrier observations, an estimate of the channel taps is obtained, from which the channel response at the intermediate data carriers can be computed straightforwardly. In contrast with the interpolation approach, the sparse channel model approach does not suffer from a performance error floor [1]. The performance of the sparse channel pilot aided channel estimation (PACE), however, depends on the selection of the pilot carrier positions. The first ones that tried to optimize the positions of the pilot carriers were Nehi and Cioffi in [8]. However, they restricted their attention to the case where the number of pilot carriers $M$ divides the total number of carriers $N$, resulting in equispaced pilots. The same results were obtained by [9] and [10] for integer $N / M$. When $N / M$ is not integer, the uniform, equispaced pilot carrier placement is no longer the optimal placement: to the contrary, the performance can become very bad. In [10], the authors proposed an ad hoc solution for the general case when $N / M$ is not integer. The resulting sub-optimal quasi-uniform pilot carrier placement turns out to have satisfactory performance. Since then, other researchers have tried to find the best pilot carrier placement for the general case. In [1], the authors maximize the distance between adjacent pilot carriers; this distribution generalizes the quasi-uniform distribution from [10] for the case when guard bands are present. In [11], the authors minimize the coherence in partial DFT matrices to obtain the pilot carrier positions. In [12], the pilot carrier placement is selected based on the minimization of a function of the Cramer-Rao lower bound (CRB). In [13], the authors propose a cubic parametrization of the pilot carriers together with convex optimization. In [14], the authors propose a pilot design based on the $l_{\infty}$ norm of the MSE of the channel/symbol estimate to jointly optimize a preamble 
and the pilot positions. Common to al these techniques is the sub-optimality: none of proposed techniques really minimizes the MSE of the channel estimate. Further, except for [1], [13] and [14], that consider the presence of guard bands at the edges on the frequency band, none of the other papers takes into account guard bands that are distributed over the whole frequency band.

Note that the aforementioned papers all deal with OFDM using the cyclic prefix (CP) as the guard interval. To our best knowledge, no research has been done on the effect of the pilot carrier placement on multicarrier systems using other guard interval techniques as e.g. zero padding (ZP) or known symbol padding (KSP) (also known as time-domain synchronous (TDS) OFDM) [15]-[17], and neither on the effect of guard bands due to the presence of primary users in a cognitive radio system. In this paper, we consider the effect of the guard interval type and the presence of guard bands on the pilot carrier placement that minimizes the MSE. As this optimization problem is an integer combinatorial optimization problem, it is NP-hard. In [18], we have proposed two heuristic algorithms, belonging to the class of linear programming algorithms, to solve the pilot carrier placement optimization problem for CP-OFDM with acceptable computational complexity. To investigate the optimality of these two algorithms, in [18] the output of both algorithms is compared with the results of an exhaustive search. Both algorithms were able to find in the vast majority of the cases (in more than $95 \%$ and $85 \%$ of the cases, respectively) the global optimum, and in the cases where the global optimum was not found, the resulting MSE was very close to (less than $0.1 \%$ above the) minimum value for the MSE. From the two algorithms proposed in [18], the one based on the hill climbing technique [19] is the simplest to work with and has the lowest computational complexity. The complexity is a major issue if we want to use the algorithm in a cognitive radio scenario where the available frequency bands are dynamic. Therefore, we opted to adapt the second algorithm to analyze the optimization of the pilot carrier placement in this paper. The questions we want to answer in this paper are: 1) Does the optimal pilot carrier placement depend on the guard interval type? 2) Does the performance of the channel estimation (strongly) depend on the used guard interval type? 3) What is the effect of the presence of guard bands on the optimal pilot carrier placement? 4) Is the heuristic algorithm that computes the optimal pilot carrier positions fast enough to be used in a cognitive radio scenario, where the carriers that can be used by the secondary users are constantly changing? Based on the numerical results in this paper, we aim to define some simple rules of thumb to select the pilot carrier positions.

\section{THE MulticARrier SyStem}

In this paper, we restrict our attention to the study of OFDM systems. However, the results can straightforwardly be used for other types of multicarrier systems as e.g. OFDMA, MC-CDMA, MC-DS-CDMA, MIMO-OFDM, ... In the following, we assume that the channel impulse response varies slowly as compared to the OFDM symbol duration, such that we can use the block fading model. Hence, during the observed OFDM symbol, the channel is fixed. We model the channel as a tapped delay line $h(k), k=0, \ldots, L-1$. We assume that the guard interval duration exceeds the length of the channel impulse response, i.e. $\nu \geq L-1$. To estimate the channel, we assume that $M$ known symbols are inserted in the frequency domain at carrier positions $n_{\ell}, \ell=1, \ldots, M$, i.e. the pilot carriers. At the receiver, we use the discrete Fourier transform (DFT) outputs corresponding to these pilot carrier positions as an observation for ML data aided channel estimation. In the following, we will show that for the three guard interval techniques, the observation vector can be written as

$$
\mathbf{y}=\mathbf{A h}+\boldsymbol{\omega}
$$

where $\mathbf{h}=[h(0) \ldots h(L-1)]^{T}$ is the channel vector, the matrix $\mathbf{A}(M \times L)$ depends on the inserted pilots, and $\boldsymbol{\omega}$ is zero mean Gaussian distributed with autocorrelation matrix $\mathbf{R}_{\omega}$, i.e. $\boldsymbol{\omega} \sim$ $N\left(\mathbf{0}, \mathbf{R}_{\boldsymbol{\omega}}\right)$. Based on this observation vector, the ML estimate of the (deterministic) channel vector is defined as [21]

$$
\tilde{\mathbf{h}}_{M L}=\arg \max _{\mathbf{h}}(\ln ) p(\mathbf{y} \mid \mathbf{h})
$$

where $p(\mathbf{y} \mid \mathbf{h}) \sim N\left(\mathbf{A h}, \mathbf{R}_{\omega}\right)$. In case $\mathbf{R}_{\boldsymbol{\omega}}$ is independent of $\mathbf{h}$, and $\mathbf{A}^{H} \mathbf{R}_{\omega}^{-1} \mathbf{A}$ is invertible, the ML estimate is given by

$$
\tilde{\mathbf{h}}_{M L}=\left(\mathbf{A}^{H} \mathbf{R}_{\omega}^{-1} \mathbf{A}\right)^{-1} \mathbf{A}^{H} \mathbf{R}_{\omega}^{-1} \mathbf{h}
$$

and the MSE of the estimation yields

$$
M S E=E\left[\left\|\tilde{\mathbf{h}}_{M L}-\mathbf{h}\right\|^{2}\right]=\operatorname{trace}\left(\left(\mathbf{A}^{H} \mathbf{R}_{\omega}^{-1} \mathbf{A}\right)^{-1}\right) .
$$

It can be verified that under the above conditions, the MSE (4) coincides with the Cramer-Rao lower bound [21], i.e. the ML estimate is a minimum variance unbiased (MVU) estimate.

The MSE of the channel estimate depends on the positions of the pilot carriers through the matrix A. Simulations show that this MSE can strongly vary with the pilot carrier positions, and when these pilot carriers are badly chosen, the resulting MSE can be very high. Such a high MSE will have an adverse effect on the overall system performance, and must therefore be avoided. Therefore, in this paper, we look for the positions of the pilot carriers that minimize the MSE.

\section{A. $C P-O F D M$}

In CP-OFDM, the transmitted symbols are converted to the time domain using an inverse DFT, and the last $\nu$ samples of this IDFT are copied and placed in front of the OFDM symbol (see Fig. 1(a)). At the receiver, the samples from the guard interval are removed and the remaining $N$ samples of the OFDM block are converted to the frequency domain. The outputs of the DFT corresponding to the $M$ pilot carrier positions can easily be derived as

$$
\mathbf{y}_{C P}=\mathbf{A}_{C P} \mathbf{h}+\mathbf{w}_{C P}
$$

where $\mathbf{A}_{C P}(\ell, k)=\sqrt{\frac{N}{N+\nu}} a_{p}(\ell) e^{-j 2 \pi \frac{k n_{\ell}}{N}}, \ell=1, \ldots, M$, $k=0, \ldots, L-1, a_{p}(\ell)$ are the pilot symbols, $\sqrt{\frac{N}{N+\nu}}$ is a normalization factor for the energy such that the energy per transmitted (data or pilot) symbol equals $E_{s}$, and $\mathbf{w}_{C P}$ is the additive Gaussian noise. It can be verified that the autocorrelation matrix of the noise is given by $\mathbf{R}_{C P}=N_{0} \mathbf{I}_{M}$ with $\mathbf{I}_{M}$ the $M \times M$ 
a)

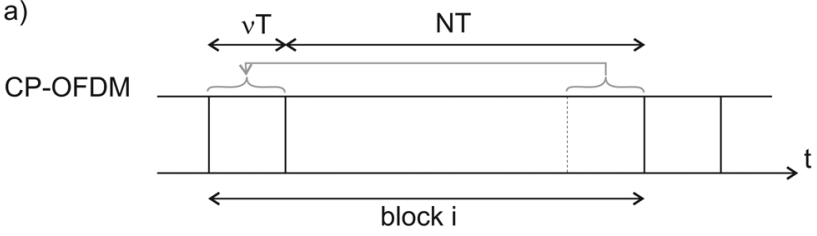

ZP-OFDM

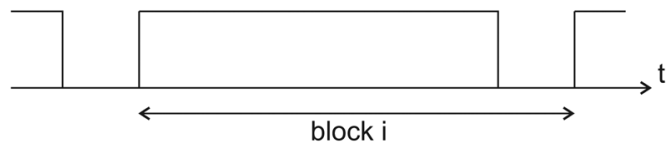

KSP-OFDM

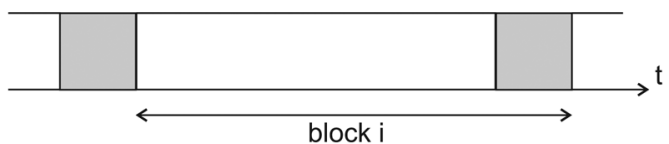

b)
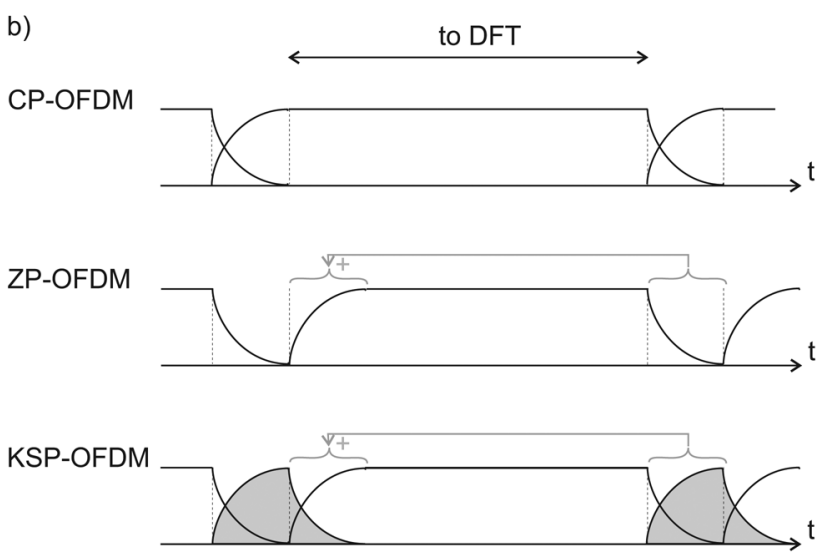

Fig. 1. Time-domain signal.

identity matrix, i.e. the additive noise is $\mathrm{AWGN}$, and $N_{0}$ is the noise variance. As such, the chosen observation complies with the observation model (1). The MSE of the channel estimate (4) for CP-OFDM is a function of the matrix $\frac{1}{N_{0}} \mathbf{A}_{C P}^{H} \mathbf{A}_{C P}$, where

$$
\frac{1}{N_{0}}\left(\mathbf{A}_{C P}^{H} \mathbf{A}_{C P}\right)_{k, k^{\prime}}=\frac{E_{s}}{N_{0}} \frac{N}{N+\nu} \sum_{\ell=1}^{M} e^{j 2 \pi \frac{\left(k-k^{\prime}\right) n_{\ell}}{N}}
$$

and where it is assumed that $\left|a_{p}(\ell)\right|^{2}=E_{s}$.

In [18], it is shown that the MSE (6) is lower bounded by

$$
M S E_{L B}=\frac{N}{N+\nu} \frac{L}{M}\left(\frac{E_{s}}{N_{0}}\right)^{-1} .
$$

This lower bound is reached when the matrix $\mathbf{A}_{C P}^{H} \mathbf{A}_{C P}$ is a diagonal matrix. For the case that $M$ divides $N$, this is achieved when the pilot carriers are equidistantly spaced. Hence, in this special case, the optimal distribution of the pilot carriers is known. However, for the general case, when $M$ does not divide $N$, the optimal solution is not known. The optimization problem at hand is an integer combinatorial optimization problem, which is NP-hard. This implies that an exhaustive search over all possible positions for the pilot carriers will be prohibitively computationally complex. Therefore, in the following section, a heuristic algorithm will be considered to find a (close to optimal) solution.

\section{B. ZP-OFDM}

In ZP-OFDM, similarly as in CP-OFDM, the transmitted symbols are modulated on the carriers using an IDFT. However, in contrast to CP-OFDM, in ZP-OFDM a guard interval containing $\nu$ zeroes is added after the OFDM symbol (see Fig. 1(a)). At the receiver, the $\nu$ samples of the guard interval are added to the $\nu$ first samples of the OFDM symbol (see Fig. 1(b)) in order to restore the orthogonality between the carriers, and the resulting $N$ samples are converted to the frequency domain. The observations at the $M$ pilot carrier positions are given by

$$
\mathbf{y}_{Z P}=\mathbf{A}_{Z P} \mathbf{h}+\mathbf{w}_{Z P}
$$

where $\mathbf{A}_{Z P}=\sqrt{\frac{N+\nu}{N}} \mathbf{A}_{C P}$ and the additive noise $\mathbf{w}_{Z P}$ has autocorrelation matrix $\mathbf{R}_{Z P}$ [16]:

$$
\left(\mathbf{R}_{Z P}\right)_{\ell, \ell^{\prime}}=N_{0}\left(\delta_{\ell, \ell^{\prime}}+\frac{1}{N} \sum_{k=0}^{\nu-1} e^{j 2 \pi \frac{\left(n_{\ell}-n_{\ell^{\prime}}\right) k}{N}}\right) .
$$

Hence, also the ZP-OFDM system complies with the model (1). The MSE of the channel estimate is determined by the matrix product $\mathbf{A}_{Z P}^{H} \mathbf{R}_{Z P}^{-1} \mathbf{A}_{Z P}$. Taking into account that for $\nu \ll N$, the non-diagonal elements of $\mathbf{R}_{Z P}$ are very small as compared to the diagonal elements (see Fig. 2), we can approximate this matrix product as

$$
\begin{aligned}
& \left(\mathbf{A}_{Z P}^{H} \mathbf{R}_{Z P}^{-1} \mathbf{A}_{Z P}\right)_{k, k^{\prime}} \\
& =\sum_{\ell, \ell^{\prime}=1}^{M} a_{p}^{*}(\ell) a_{p}\left(\ell^{\prime}\right)\left(\mathbf{R}_{Z P}^{-1}\right)_{\ell, \ell^{\prime}} e^{j 2 \pi \frac{k n_{\ell}-k^{\prime} n_{\ell^{\prime}}}{N}} \\
& \approx \frac{1}{N_{0}} \frac{N}{N+\nu} \sum_{\ell=1}^{M}\left|a_{p}(\ell)\right|^{2} e^{j 2 \pi\left(k-k^{\prime}\right) n_{\ell} N} \\
& =\frac{E_{s}}{N_{0}} \frac{N}{N+\nu} \sum_{\ell=1}^{M} e^{j 2 \pi \frac{\left(k-k^{\prime}\right) n_{\ell}}{N}}
\end{aligned}
$$

where $\mathbf{R}_{Z P} \approx N_{0} \frac{N+\nu}{N} \mathbf{I}_{M}$ and $\left|a_{p}(\ell)\right|^{2}=E_{s}$. Hence, using this approximation, the MSE for ZP-OFDM is equal to that of CP-OFDM (6). Based on (10), we expect that the effect of the pilot carrier values on the MSE will be small when $\nu / N$ is sufficiently small. However, for 'large' $\nu / N$, the approximation in (10) will not be valid, such that the specific values of the pilot symbols will start to play a role.

\section{KSP-OFDM}

In KSP-OFDM, a guard interval of $\nu$ known samples-also called the time domain pilots - is added after the OFDM time domain symbol that was generated with an IDFT (see Fig. 1(a)). At the receiver, similarly as for ZP-OFDM, the samples from the guard interval are added to the $\nu$ first samples of the OFDM symbol to restore the orthogonality between the carriers (see Fig. 1(b)). The $M$ outputs of the DFT corresponding to the frequency domain pilots are given by

$$
\mathbf{y}_{K S P}=\mathbf{A}_{K S P} \mathbf{h}+\mathbf{w}_{K S P}
$$




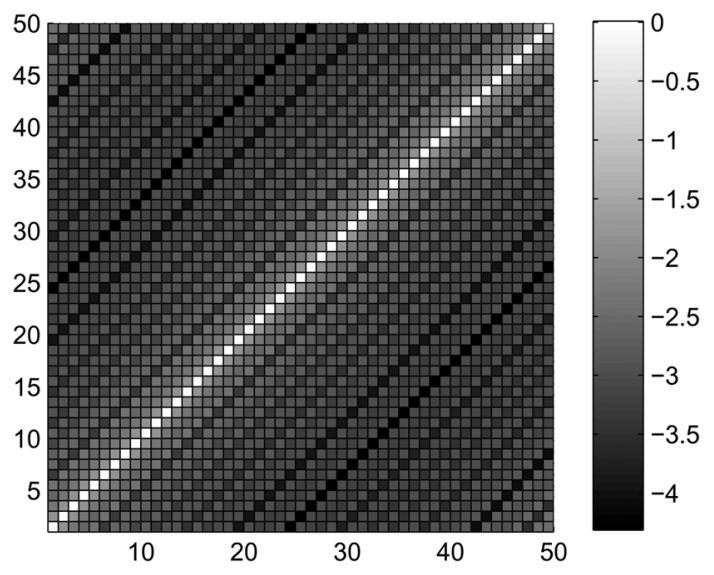

Fig. 2. The autocorrelation matrix: $\log _{10}\left|\left(\mathbf{R}_{Z P}\right)_{k, k^{\prime}}\right|$ with $N=1024, M=$ $50, \nu=20$ and uniform pilot carrier placement $n_{\ell}=\ell \Delta$ with $\Delta=20$.

where $\mathbf{A}_{K S P}=\mathbf{A}_{C P}+\tilde{\mathbf{A}}_{K S P}$, with $\mathbf{A}_{C P}$ the contribution from the frequency domain pilots, which is the same as for CP-OFDM, and $\tilde{\mathbf{A}}_{K S P}$ the contribution from the time domain pilots [16]:

$$
\tilde{\mathbf{A}}_{K S P}(\ell, k)=\sqrt{\frac{N}{N+\nu}} \frac{1}{\sqrt{N}} \sum_{m=0}^{\nu-1} e^{-j 2 \pi \frac{m n_{\ell}}{N}} a_{g}\left(|m-k|_{\nu}\right)
$$

where $a_{g}(m)$ are the known samples from the guard interval, and $|x|_{\nu}$ is the modulo- $\nu$ reduction of $x$ yielding a result in the interval $[0, \nu[$. The autocorrelation matrix of the additive noise equals $\mathbf{R}_{K S P}=\mathbf{R}_{Z P}$. So, also the KSP-OFDM system complies with (1).

The MSE of the channel estimate is determined by the matrix product $\mathbf{A}_{K S P}^{H} \mathbf{R}_{K S P}^{-1} \mathbf{A}_{K S P}$. Similarly as for ZP-OFDM, we approximate the autocorrelation matrix, for $\nu \ll N$, by $\mathbf{R}_{K S P} \approx N_{0} \frac{N+\nu}{N} \mathbf{I}_{M}$. Hence, $\mathbf{A}_{K S P}^{H} \mathbf{R}_{K S P}^{-1} \mathbf{A}_{K S P}$ reduces to $\frac{1}{N_{0}} \frac{N}{N+\nu} \mathbf{A}_{K S P}^{H} \mathbf{A}_{K S P}$. Further, taking into account that $\mathbf{A}_{K S P}=\mathbf{A}_{C P}+\tilde{\mathbf{A}}_{K S P}$, this product can be decomposed as

$$
\begin{aligned}
\mathbf{A}_{K S P}^{H} \mathbf{A}_{K S P}=\mathbf{A}_{C P}^{H} \mathbf{A}_{C P}+\mathbf{A}_{C P}^{H} \tilde{\mathbf{A}}_{K S P} & \\
& +\tilde{\mathbf{A}}_{K S P}^{H} \mathbf{A}_{C P}+\tilde{\mathbf{A}}_{K S P}^{H} \tilde{\mathbf{A}}_{K S P} .
\end{aligned}
$$

Assuming the time domain pilots are uncorrelated with the frequency domain pilots, i.e. $E\left[a_{g}^{*}(k) a_{p}(\ell)\right]=0$, we approximate the cross products by $\mathbf{A}_{C P}^{H} \tilde{\mathbf{A}}_{K S P} \approx \mathbf{0} \approx \tilde{\mathbf{A}}_{K S P}^{H} \mathbf{A}_{C P}$. The product $\tilde{\mathbf{A}}_{K S P}^{H} \tilde{\mathbf{A}}_{K S P}$ is given by

$$
\begin{aligned}
\left(\tilde{\mathbf{A}}_{K S P}^{H} \tilde{\mathbf{A}}_{K S P}\right)_{k, k^{\prime}} \\
=\frac{N}{N+\nu} \frac{1}{N} \sum_{\ell=1}^{M} \sum_{m, m^{\prime}=0}^{\nu-1} a_{g}^{*}\left(|m-k|_{\nu}\right) a_{g}\left(\left|m^{\prime}-k^{\prime}\right|_{\nu}\right) \\
\quad \cdot e^{j 2 \pi \frac{n_{\ell}\left(m-m^{\prime}\right)}{N}} \\
\approx \frac{N}{N+\nu} \frac{1}{N} \sum_{\ell=1}^{M} \sum_{m, m^{\prime}=0}^{\nu-1} E_{s} \delta_{m-k, m^{\prime}-k^{\prime}} e^{j 2 \pi \frac{n_{\ell}\left(m-m^{\prime}\right)}{N}} \\
=E_{s} \frac{N}{N+\nu} \frac{\nu-\left|k-k^{\prime}\right|}{N} \sum_{\ell=1}^{M} e^{j 2 \pi \frac{\left(k-k^{\prime}\right) n_{\ell}}{N}}
\end{aligned}
$$

where we have assumed in the second line that the pilots in the time domain are uncorrelated, i.e. $E\left[a_{g}^{*}(k) a_{g}\left(k^{\prime}\right)\right]=E_{s} \delta_{k, k^{\prime}}$. Taking into account that $\mathbf{A}_{C P}^{H} \mathbf{A}_{C P}$ is given in (6), it follows that the matrix product $\mathbf{A}_{K S P}^{H} \mathbf{R}_{K S P}^{-1} \mathbf{A}_{K S P}$ can be approximated by

$$
\begin{aligned}
& \left(\mathbf{A}_{K S P}^{H} \mathbf{R}_{K S P}^{-1} \mathbf{A}_{K S P}\right)_{k, k^{\prime}} \\
& \approx \frac{E_{s}}{N_{0}} \frac{N}{N+\nu} \frac{N+\nu-\left|k-k^{\prime}\right|}{N} \sum_{\ell=1}^{M} e^{j 2 \pi \frac{\left(k-k^{\prime}\right) n_{\ell}}{N}} \\
& \approx E_{s} \approx N_{0} \frac{N}{N+\nu} \sum_{\ell=1}^{M} e^{j 2 \pi \frac{\left(k-k^{\prime}\right) n_{\ell}}{N}}
\end{aligned}
$$

where in the second line, $N+\nu-\left|k-k^{\prime}\right|$ is approximated by $N+\nu$. This can be done as $k$ and $k^{\prime}$ range from 0 to $L-1$, with $L-1 \leq \nu \ll N$, such that $\left|k-k^{\prime}\right|$ will be small as compared to $N+\nu$. From the approximations made to obtain the last line in (15), we can conclude that when $\nu / N$ is small, the effect of both the values of the time domain pilots as the frequency domain pilots will be small. The former can be explained as, when $\nu / N$ is small, the factor $\frac{N+\nu-\left|k-k^{\prime}\right|}{N}$ in the last line of (14) will be close to one, such that the contribution $\tilde{\mathbf{A}}_{K S P}^{H} \tilde{\mathbf{A}}_{K S P}$ from the time domain pilots will be small as compared to the contribution $\mathbf{A}_{C P}^{H} \mathbf{A}_{C P}$ from the frequency domain pilots, and the latter by the fact that when $\nu / N$ is small, the matrix $\mathbf{R}_{K S P}$ will be very close to a diagonal matrix, similarly as for ZP-OFDM. On the other hand, when $\nu / N$ becomes too large, we expect that the values of both the time domain as the frequency domain pilots will play a role.

Note that in the above approximations, we have not made any assumption on the used pilot carrier placement or the presence of guard bands. As such these generic conclusions will be valid for any pilot carrier placement and if guard bands are present. Using these approximations, i.e. on the autocorrelation matrix and on the pilot statistics, we obtain for KSP-OFDM the same expression for the MSE as for CP-OFDM and ZP-OFDM. Hence, at first sight this indicates that if the approximations are valid (i.e. $\nu / N$ small), the pilot carrier placement that minimizes the MSE of the channel estimate will be essentially independent of the used guard interval technique and the MSE performance for the three guard interval techniques will be very similar. In the remainder of the paper, we will use the correct expressions for the MSE to generate the numerical results, but we will use the approximated expressions to explain the similarities and differences between the results for the different guard interval techniques.

\section{The Optimization Problem}

In the previous section, we have derived the exact and approximated MSE for the three guard interval types. We observe that the MSE for the CP-OFDM system is independent of the pilot symbols, whereas for the other two guard interval types, the MSE (without approximations) is a function of the values of the pilot symbols. In this paper, we consider the heuristic algorithm presented in [18] that was developed to find the optimal pilot carrier positions for CP-OFDM. We extend the algorithm to optimize the MSE (without approximations) for the 
TABLE I

HEURISTIC ALGORITHM

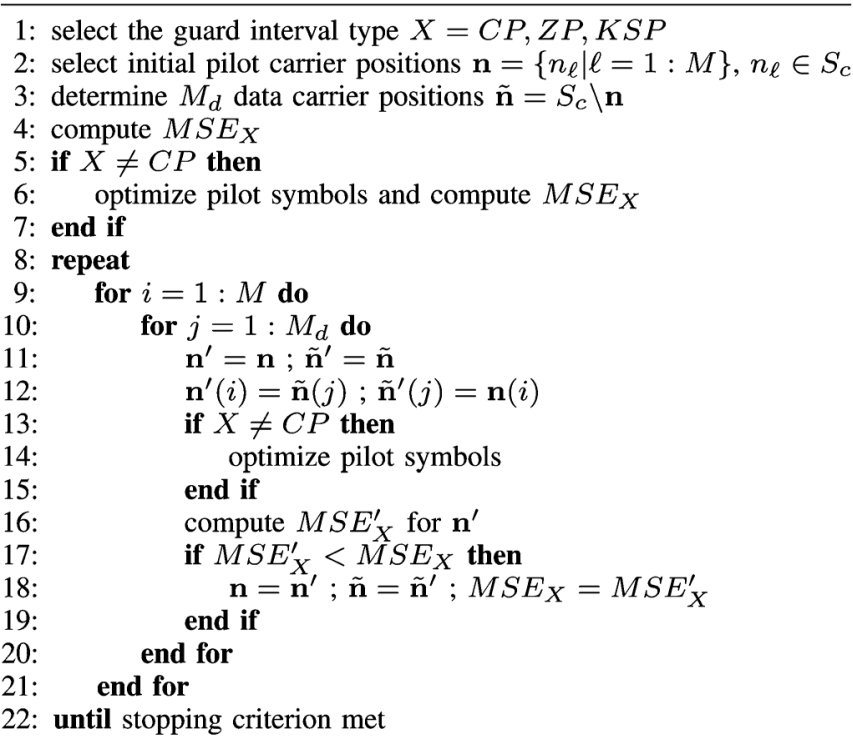

three guard interval types. The modified algorithm also takes into account the optimization of the pilot symbol values. In this paper, we restrict our attention to pilot symbols belonging to the QPSK alphabet. However, the algorithm can easily be extended to pilot symbols belonging to other alphabets.

The heuristic algorithm that we consider is based on the hill climbing technique [20], which is a local search algorithm suitable for integer combinatorial optimization problems. It starts from an initial choice for the parameters to be optimized, i.e. the pilot carrier positions, and looks in the neighborhood if there is a better solution by incrementally changing the parameters. The main drawback of this technique is that if there are multiple local optima, it converges to a local optimum, and not to the global optimum. Hence, a good initial estimate of the parameters is necessary to be able to find the global optimum. In [18], it is shown for CP-OFDM, that a good initial choice for the pilot carrier positions is the maximum distance distribution [1], that maximizes the distance between the pilot carrier positions taking into account guard bands at the edges of the frequency band. We adapt the maximum distance distribution from [1] to also take into account guard bands that are present in the spectrum to cope with primary users. The heuristic algorithm was shown in [18] to converge in the majority of the cases to the global minimum obtained with an exhaustive search. Hence, in this paper, we will use this maximum distance distribution as the initial setting for our algorithm.

The algorithm is shown in Table I. The set $S_{c}$ is the set of possible carrier positions (excluding guard band carriers). The initial settings for the pilot carrier positions are determined by the maximum distance distribution. Then, the pilot symbol values are optimized for these pilot carrier positions. In lines 9-21 of the algorithm, we systematically switch the data carrier positions with the pilot carrier positions. This is achieved with the two loops in lines 9-10. The outer loop in line 9 examines the $M$ pilot carrier positions one by one. Each of these pilot carrier positions is switched-one by one-with all $M_{d}$ data carrier positions in the inner loop (line 10). For example, first $\mathbf{n}(1)$ is switched with data carrier position $\tilde{\mathbf{n}}(j), j=1: M_{d}$, and for the resulting set of pilot carrier positions, the pilot symbol values are optimized. We check if the resulting MSE is smaller than the current best MSE. If the new MSE is smaller, the switch in the positions is kept, and otherwise, the data carrier position and the pilot carrier position are restored to the previous state. Hence, in lines 9-21, $M \cdot M_{d}$ of these switches are tested. To improve the convergence of the algorithm, we can iteratively execute lines 9-21 of the algorithm, where the output of the previous run is taken as the initial setting for the next run. In our simulations, we have always iterated the algorithm until no further improvement was achieved, i.e. until $M S E_{i-1}=M S E_{i}$, where $i$ is the iteration index. Although in theory this could take infinitely long, the proposed algorithm has a good convergence: in practice, we found that the algorithm typically converges within a limited number of iterations. ${ }^{1}$ Of course, we could use a different stopping criterion as e.g. when $M S E_{i-1}-M S E_{i} \leq$ threshold, i.e. when the difference in the MSE value in successive iterations becomes smaller than a predefined threshold, or after a fixed number of iterations. In the numerical results, we will discuss further on the convergence and the stopping criterion.

\section{NumERICAL RESUlTS}

\section{A. Effect of the Pilot Carrier Symbols}

First we evaluate the effect of the pilot symbol values on the MSE performance for the three guard interval types without optimizing the pilot carrier positions. From our simulations, it followed that as expected, similar results on the optimization of the pilot symbols are obtained irrespective of the used pilot carrier placement or the presence of guard bands. Therefore, in this section we present the results for the case where the pilot carriers are selected according to the maximum distance (MD) distribution from [1], and all carriers can be used as pilot carriers, i.e. no guard bands are present. The optimal pilot symbol values are obtained as follows. We randomly generate 1000 QPSK pilot carrier symbol sequences, and select the best sequence resulting in the lowest MSE. For KSP-OFDM, for the best pilot carrier sequence, we then randomly generate 1000 QPSK guard interval pilot sequences, and select again the sequence resulting in the lowest MSE. This simulation is repeated 100 times. In Figs. 3(a) and 3(b), the average of the lowest MSE and its standard deviation over the 100 experiments is shown. We considered three cases. In the first case, the pilot carrier symbols are selected to optimize the MSE of ZP-OFDM, and the resulting pilot carrier sequence is used to compute the MSE for the three guard interval types. In the second case, the pilot carrier symbols are optimized for KSP-OFDM, and similarly as in case 1, the resulting symbols are used for the other guard interval techniques. In the third case, the pilot carrier symbols are not optimized, but randomly selected. In all cases, for KSP-OFDM, the guard interval pilot symbols are selected as the best out of 1000 randomly generated sequences.

\footnotetext{
${ }^{1}$ In only a very limited amount of cases we investigated, the maximum number of iterations needed to obtain $M S E_{i-1}=M S E_{i}$ exceeded 10. Most of the time, less than 5 iterations were needed for the algorithm to converge.
} 


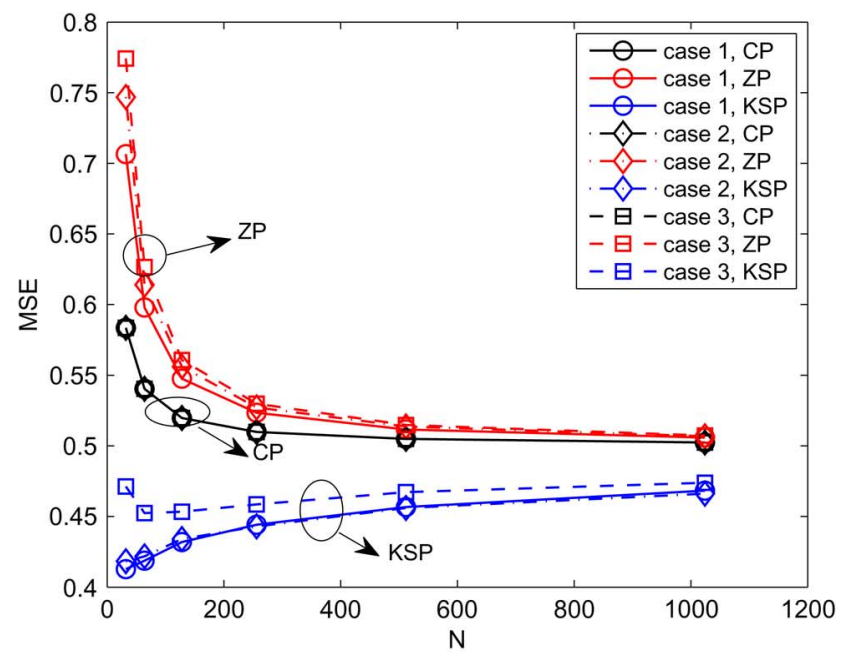

(a)

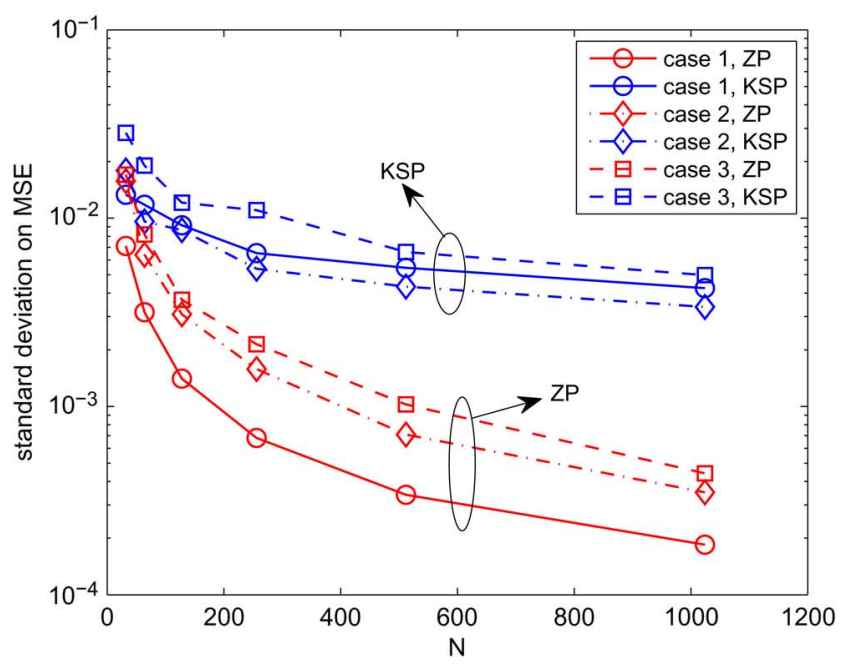

(b)

Fig. 3. The MSE (a) and standard deviation of the MSE (b) for the different guard interval types (case $1=$ pilot symbols optimized for ZP, case $2=$ pilot symbols optimized for KSP, case 3 : no optimization pilot symbols), $M=10$, $L=5, \nu=L$, no guard band, maximum distance distribution.

First we observe that, as expected, the MSE of CP-OFDM is independent of the used pilot carrier symbols. Further we notice that when the pilot carrier symbols are not optimized (case 3), the resulting MSE for ZP-OFDM and KSP-OFDM is worse than for the two other cases. For ZP-OFDM, the difference is however small, whereas for KSP-OFDM the difference is larger. Especially for small values of the total number of carriers $N$, the effect of the optimization of the pilot carrier symbols is visible, whereas for large $N$, the difference becomes small. This is in line with the conclusions from Section II: when $\nu / N$ is small, the effect of not optimizing the pilot symbols will be negligible. When $\nu / N$ becomes larger, the values of the pilot symbol will play a role. For ZP-OFDM, we only have the frequency domain pilots, whereas in KSP-OFDM, we have both time and frequency domain pilots, so we expect the effect of optimizing the pilot symbol values in KSP-OFDM is larger.

In the paper, we have shown that the MSE for CP-OFDM is an approximation for the MSE for both ZP-OFDM and KSP-OFDM. From the figure, it follows that the true MSE for
ZP-OFDM (KSP-OFDM) is larger (smaller) than its approximation, but that for increasing $N$ the difference reduces. This latter effect is explained by the fact that for a given $\nu$, increasing $N$ will lead to a smaller $\nu / N$, such that the approximations will become valid. The former effect could also be expected: for ZP-OFDM, because of the non-diagonal nature of the autocorrelation matrix $\mathbf{R}_{Z P}$, we expect the true MSE for ZP-OFDM to be slightly larger than for CP-OFDM, and for KSP-OFDM, as we have more pilots (i.e. the guard interval pilots) than in CP-OFDM, there is more information present that can be used to estimate the channel, resulting in a lower MSE.

Secondly, we look at the effect of the optimization of the pilot carrier symbols. When we compare the MSE results for case 2 and 3, we observe in Fig. 3(a) that for KSP-OFDM, there is virtually no difference between the two cases, whereas for ZP-OFDM, case 1 gives slightly better performance. Taking into account the standard deviation of the MSE in Fig. 3(b), we can conclude that case 1 , where the pilot carrier symbols are optimized for ZP-OFDM, is virtually optimal for both ZP-OFDM and KSP-OFDM. This implies that the optimal values for the pilot carrier symbols are mainly determined by the non-diagonal nature of the autocorrelation matrix. As a conclusion, we do not have to take into account the values of the guard interval pilots for KSP-OFDM when optimizing the values of the pilot carrier symbols. This will reduce the computational complexity of the optimization. Hence, in the following sections, we will use the ZP-OFDM MSE metric to optimize the pilot carrier symbol values for both ZP-OFDM and KSP-OFDM.

\section{B. Optimization of the Pilot Carrier Positions}

In the previous section, we did not consider the presence of guard bands where the pilot carriers cannot be placed. In Fig. 4, we consider three types of guard band distributions that are used in the following simulations. In case (a), there are only guard bands of length $\gamma$ at the edges of the OFDM frequency band to separate the OFDM signal from transmissions in adjacent frequency bands; hence there are $2 \gamma$ forbidden carrier positions and $N-2 \gamma$ carriers that can be used for data and pilot carriers. In case (b), besides the guard bands of case (a), we also consider the DC carrier as not allowed for data or pilot transmission. Hence, there are $2 \gamma+1$ forbidden carriers. In case (c), besides the guard bands of case (a), we also consider a guard band of length $\alpha$, starting at position $n_{\alpha}$, so $2 \gamma+\alpha$ carriers are not allowed for pilots or data. This last case e.g. corresponds to a cognitive radio system where in the band $\alpha$ a primary user is transmitting, and to avoid interference with the primary user, the secondary OFDM system is not allowed to transmit in this frequency band.

First of all, we will examine the effect of the number of iterations of the heuristic algorithm. We use the guard band distribution from Fig. 4(a). In Fig. 5, the MSE at the $k$ th iteration is compared with the minimum MSE, i.e. $M S E_{\min }$, that can be obtained with the heuristic algorithm: ${ }^{2}$ $\left(M S E_{k}-M S E_{\min }\right) / M S E_{\min } \cdot 100$ (in \%), where the zero-th

\footnotetext{
${ }^{2}$ In this simulation, for all considered cases, the algorithm has converged in less than five iterations, i.e., increasing the number of iterations will not result in a change in the pilot carrier positions. Hence, in this simulation, $M S E_{5}$ can be considered as the reference lower bound $M S E_{\min }$ of the MSE.
} 
(a)

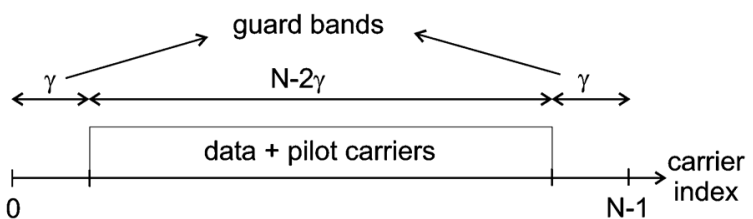

(b)

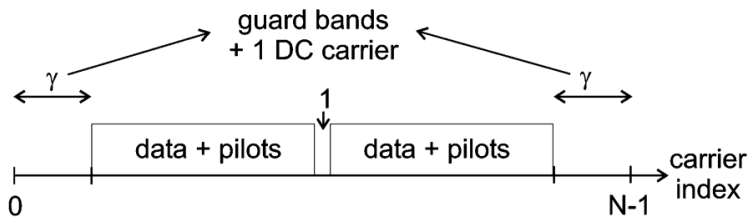

(c)

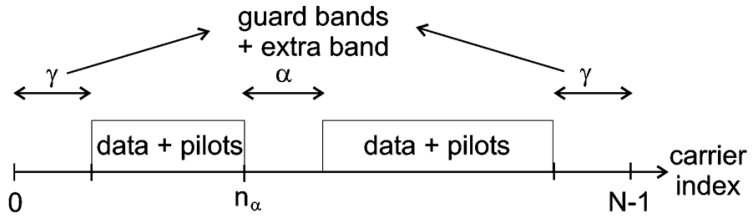

Fig. 4. Different guard band distributions.

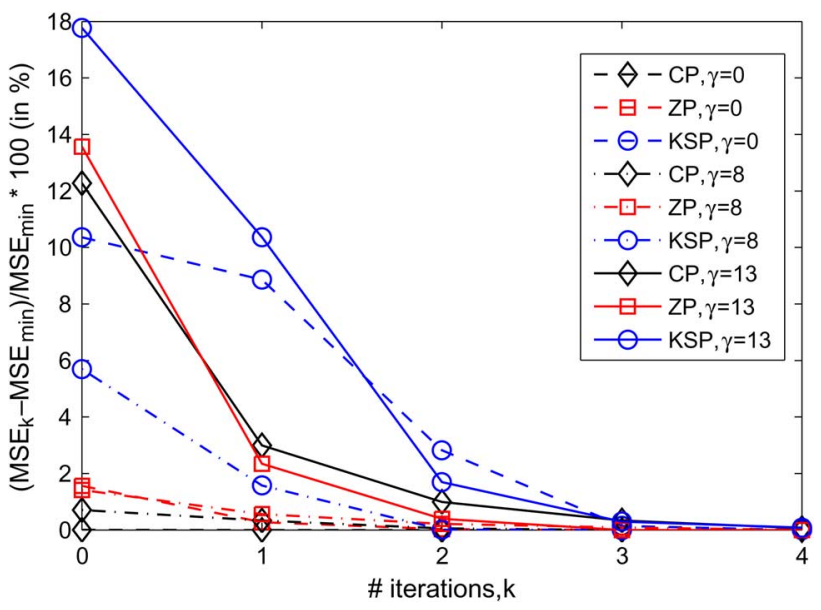

Fig. 5. The effect of the number of iterations of the heuristic algorithm $L=5$, $\nu=L, M=10, N=128$.

iteration corresponds to the maximum distance distribution for the pilot carriers, with optimized pilot symbol values. It can be observed that when the guard band length $\gamma$ increases, generally more iterations are needed to converge. Further, KSP-OFDM typically needs more iterations than the other two OFDM systems; this is caused by the interaction between the time domain and frequency domain pilots (see (13)). However, for all considered cases in this simulation, and also for all other simulations we did, 2-3 iterations were sufficient to obtain a MSE that is only $1-2 \%$ higher than the minimum value for the MSE that could be obtained with the heuristic algorithm. Other simulations, not shown in this paper due to space limitations, for other stopping criteria (e.g. until the difference between successively obtained MSE values is below a threshold, or until convergence is reached) or other system parameters have been carried out; the results for all considered cases were similar. The computational complexity of the algorithm is an issue if we want to use it in a practical cognitive radio scenario, where the available carriers are constantly changing. Hence, it is of primordial importance that in that case, the best pilot carrier placement can be computed in a very fast way, so the

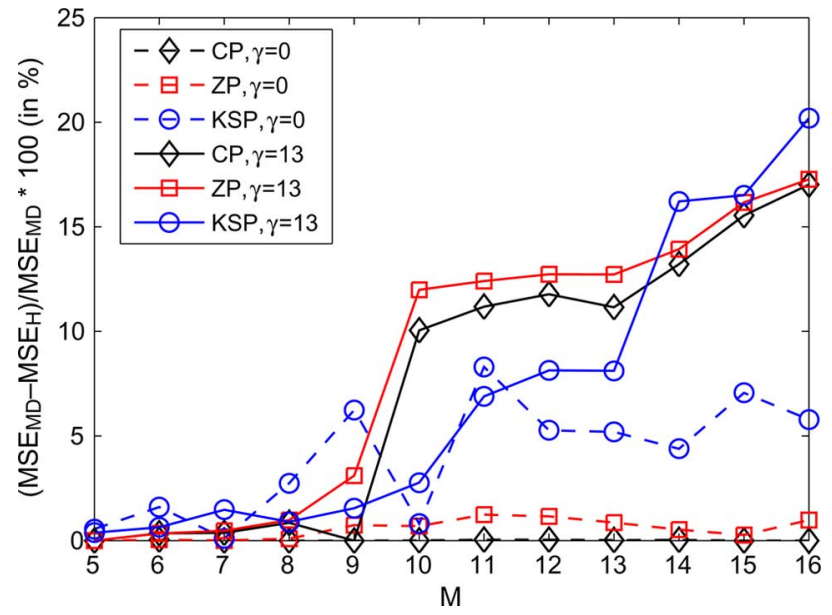

Fig. 6. Effect of the number $M$ of pilot carriers, $L=5, \nu=L, N=128$, $M S E_{M D}=\mathrm{MSE}$ for maximum distance distribution, $M S E_{H}=\mathrm{MSE}$ for heuristic algorithm with 2 iterations.

computational complexity of the algorithm must be as low as possible. As a further increase in the number of iterations will only lead to a marginal performance improvement and a linear increase in computational complexity, we suggest to limit the number of iterations to $2-3$. In the remainder of the paper, all results are obtained with 2 iterations of the heuristic algorithm.

The effect of the number $M$ of pilot carriers is shown in Fig. 6. For an increasing number of pilot carriers, generally the gain in using the heuristic algorithm to find the pilot positions compared to the maximum distance distribution increases. For the case with $\gamma=13$, one can observe a sharp increase in the gain for $M \geq 10$. This can be explained as follows. The maximum distance distribution spreads the pilot carriers as uniformly as possible over the available frequency band. If we would be allowed to place the pilot carriers over the whole frequency band, the maximum possible distance between two pilot carriers equals $\lceil N / M\rceil$, and the distance between two adjacent carriers will be $\lfloor N / M\rfloor$ or $\lceil N / M\rceil$. In [1] and [18], it is shown that for $\gamma=0$ this placement results in a MSE that is very close to the global minimum (7). However, we are not allowed to place pilots in the guard bands. As long as $\gamma<\lceil N / M\rceil$, the guard band will not strongly interfere with the pilot carrier placement: the maximum distance distribution simply places the pilot carriers around the guard band, and if necessary it slightly shifts a few pilot carriers. Simulations show that the global minimum is a broad minimum, which implies that slightly shifting some pilot carriers will result in a MSE that is still close to the global minimum. Hence, the maximum distance distribution can be considered as quasi-optimal if $\gamma<\lceil N / M\rceil$. However, when $\gamma>\lceil N / M\rceil$, a situation that is illustrated in Fig. 7, the distance between the pilot carriers decreases, and the distance between the edge pilot carriers (i.e., $2 \gamma+1),{ }^{3}$ will be quite large compared to the other pilot carrier spacings. As such, the resulting MSE will be further away from the global minimum (7), and simulations show that in this case, slightly shifting the pilot carrier positions will result in a large change in the MSE. Typically, the

\footnotetext{
${ }^{3}$ Note that the MSE is insensitive to a cyclic shift of the pilot carriers, implying that we can cyclically shift the pilot carriers and the guard band over the spectrum without having an effect on the MSE.
} 


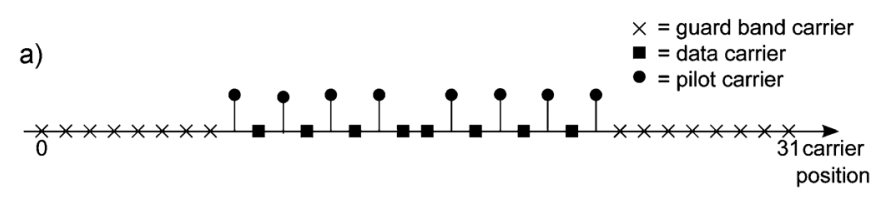

b)

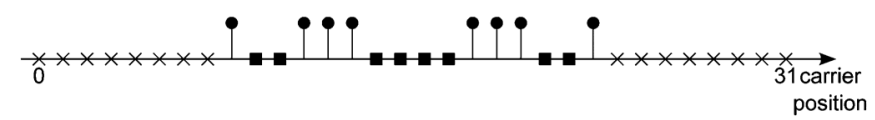

c)

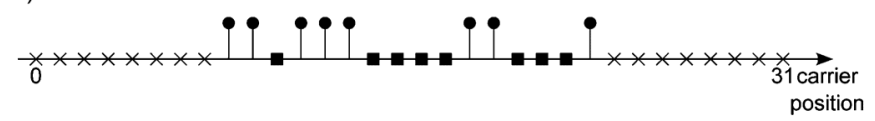

d)

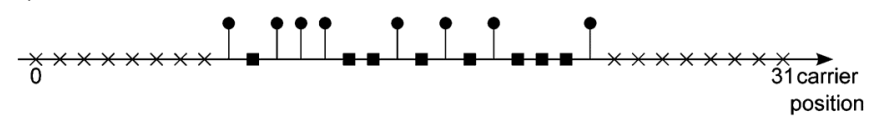

Fig. 7. Optimal pilot positions for $N=32, L=4, \nu=L, M=8, \gamma=8$.

maximum distance distribution will not be the optimal one, and the gain in optimizing the pilot carrier positions will be large.

Next, we compare the results from the heuristic algorithm with the results for the maximum distance distribution, for different values for $N$. We consider the guard band distribution (a). From our simulations (not shown in the paper), we observed that for $\gamma=0$, the performance improvement obtained with the heuristic algorithm is rather small. This indicates that when there is no guard band, the maximum distance distribution is quasi-optimal. However, when the guard band length increases, typically the gain, when using the heuristic algorithm instead of the maximum distance distribution, increases. This indicates that the maximum distance distribution is not the best choice for the pilot carrier positions when a non-negligible part of the spectrum is not available for transmission, as e.g. in cognitive radio systems. As an example, we show in Fig. 8 the gain when using the heuristic algorithm instead of the maximum distance distribution for $\gamma=8$. It can be observed that when $N$ increases, the gain in using the heuristic algorithm decreases, except for KSP case 2. This can be explained as in case $2, N / M<\gamma$, and as explained for Fig. 6, a slight shift of the pilot carriers in that case can have a large influence on the MSE. From our simulations, we have observed that this effect will mainly play a significant role for KSP-OFDM, but for CP-OFDM and ZP-OFDM, it will only have a moderate effect when $N$ increases. When we would plot the value of the MSE (instead of the relative gain) as function of $N$ a similar result as in Fig. 3(a) is obtained: the MSE values for CP, ZP and KSP-OFDM come closer to each other when $N$ increases, because the effect of the pilot symbols is reduced, as explained in the previous section for the maximum distance distribution. The simulation results also revealed that for large $N$ and $N / M>\gamma$, the pilot carrier positions delivered by the heuristic algorithm for CP-OFDM (ZP-OFDM, KSP-OFDM) also yielded essentially the lowest MSE for the other two cases. This indicates that the approximations made in Section II are valid, resulting in the same expressions for the MSE for the three guard interval techniques. However, for small values of $N$, the difference between the optimal pilot carrier placement for the different guard interval techniques can

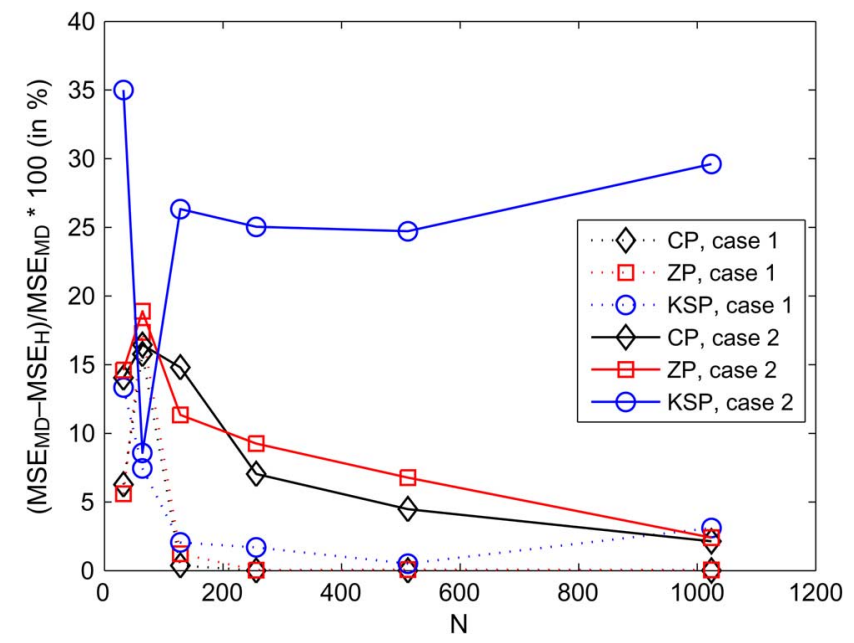

Fig. 8. Optimizing the pilot carrier positions and pilot symbol values, $\nu=L$, $M=2 L, \gamma=8, M S E_{M D}=$ MSE for maximum distance distribution, $M S E_{H}=\mathrm{MSE}$ for heuristic algorithm with 2 iterations, case 1: $L=5$, case 2: $L=\lceil 0.1 N\rceil$.

be quite large. This is illustrated in Fig. 7. Moreover, for large $N$, as the performance gain decreases, using the maximum distance distribution will be a good compromise between performance and complexity: it has a MSE that is typically only less that $10 \%$ higher than for the optimal placement, and can be obtained at no computational cost.

\section{Effect of the Guard Band Distribution}

In this section, we evaluate the influence of the distribution of the guard bands on the MSE performance. In Fig. 9, the MSE performance (normalized on the lower bound (7) on the CP-OFDM performance obtained with the heuristic algorithm is shown for five cases, i.e. case (a) and (b) from Fig. 4, and three situations for case (c) in Fig. 4 with different $n_{\alpha}$ and $\alpha$. From the figure, it is clear that the MSE is essentially independent of the considered cases: with the heuristic algorithm, the presence or position of guard bands has almost no influence on the MSE performance. This is in contrast with the maximum distance distribution, where the MSE noticeably increases for guard band distribution (c) when $\alpha$ increases: the gain of using the heuristic algorithm instead the maximum distance distribution is typically doubled for case (c) compared to cases (a) and (b). Note also that $M S E_{H} / M S E_{L B}$ is essentially independent of $M$ and close to 1 for all cases. This indicates that $M S E_{L B}$, which is proportional to $L / M$, and was only shown for CP-OFDM to be a lower bound on the MSE, is a good approximation of the MSE for the three guard interval types.

\section{CONCLUSIONS}

In this paper, we have investigated the effect of the pilot carrier positions on the performance of channel estimation in OFDM based systems. We have considered three guard interval types, cyclic prefix, zero padding and known symbol padding. Traditionally, the maximum distance distribution from [1] is used to select the positions of the pilot carriers. However, when parts of the bandwidth are not available for placing the pilot 


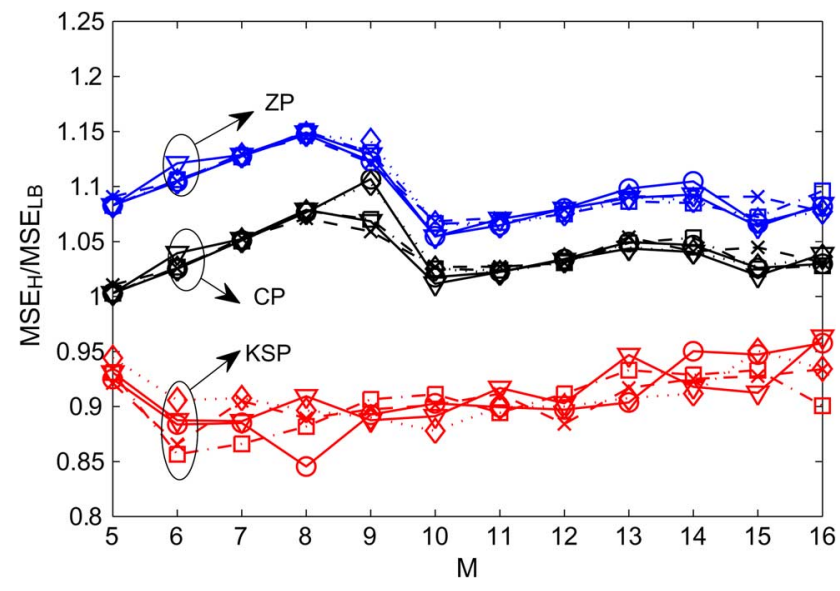

Fig. 9. Effect of the distribution of the guard bands, pilot positions selected according to the heuristic algorithm, $N=128, L=\nu=5, \gamma=13$ with as markers for the different guard band distributions: $\circ=$ case (a) from Fig. 4; $\diamond=$ case (b); $\square=$ case (c) with $n_{\alpha}=50$ and $\alpha=10 ; \times=$ case (c) with $n_{\alpha}=50$ and $\alpha=20 ;$ and $\nabla=$ case (c) with $n_{\alpha}=65$ and $\alpha=20$.

carriers, as e.g. in cognitive radio, the maximum distance distribution turns out to be not optimal, and even can give rise to high MSE.

In this paper, we have evaluated the analytical expressions for the MSE for the different guard interval techniques, and we have shown that, taking into account some approximations, the MSE performance is (approximately) the same irrespective of the used guard interval. These approximations turn out to be valid for large values of the DFT size $N$ and relatively short guard intervals $\nu / N \ll 1$. In that case, the MSE for CP-OFDM is then a small underestimation of the MSE of ZP-OFDM, and a small overestimation for the MSE for KSP-OFDM.

Another observation is that, if $N$ is sufficiently large (and for KSP-OFDM if the lengths of the guard bands are smaller than $N / M$ ), the maximum distance distribution is almost optimal. In that case, it is not necessary to apply the heuristic algorithm used in this paper: the relative gain that can be obtained is rather small at the cost of an increased computational complexity. However, when $N$ is small and the guard band lengths increase above $N / M$, it is better to use the heuristic algorithm to find the best pilot carrier positions, as it clearly outperforms the maximum distance distribution. Although in this paper, we have focussed on the ML estimator, we can easily apply the heuristic algorithm to other estimators; we only have to replace in the heuristic algorithm the MSE with the appropriate expression.

The goal of this paper was to find some answers on the sense or nonsense of trying to optimize the pilot carrier positions in cognitive radio based multicarrier systems. In the introduction, we posed the following questions, which we now can answer. Further, Table II summarizes the rules of thumb, taking into account both computational complexity and performance.

1) Does the optimal pilot carrier placement depend on the guard interval type?

When $\nu / N$ is sufficiently small and $N$ sufficiently large, the difference between the three guard interval types is small: the pilot carrier placement that is optimal for CP-OFDM will also be quasi optimal for the other guard
TABLE II

When to Use the Heuristic Algorithm AND When to OPTIMIZE THE PILOT SYMBOLS?

\begin{tabular}{|c|c|}
\hline use maximum distance distribution & if $N \geq 256$ \\
\hline optimize pilot carrier positions & only if $N / M<$ guard band width \\
\hline $\begin{array}{c}\text { optimize pilot carrier symbols } \\
\text { (ZP and KSP) }\end{array}$ & $\begin{array}{c}\text { only if } \nu / N \text { not sufficiently small } \\
\text { and } N<256\end{array}$ \\
\hline $\begin{array}{c}\text { optimize time-domain pilot values } \\
\text { (KSP) }\end{array}$ & $\begin{array}{c}\text { only if } \nu / N \text { not sufficiently small } \\
\text { and } N<64\end{array}$ \\
\hline
\end{tabular}

interval techniques. However, for smaller $N$, this will not be the case.

2) Does the performance of the channel estimation (strongly) depend of the used guard interval type?

If the DFT size is small $(N \leq 64)$, the difference in MSE for the different guard interval techniques can be quite large. However, when $N$ increases, this difference becomes smaller and smaller.

3) What is the effect of the presence of guard bands on the optimal pilot carrier placement?

As long as $N$ is sufficiently large, the maximum distance distribution will give satisfactory results. However, for smaller values of $N$, when $\gamma>N / M$, the pilot carrier positions should be optimized, otherwise the MSE performance will degrade rapidly.

4) Is the heuristic algorithm that computes the optimal pilot carrier positions fast enough to be used in a dynamic cognitive radio scenario?

In [18], it is shown that the computational complexity of the algorithm is approximately $\mathcal{O}\left(M \cdot M_{d} \cdot L^{3}\right)$ per iteration. Hence, for small $N$, where the use of the heuristic algorithm is advised, also $M, L$ and $M_{d}$ will be small, such that the computational complexity of the heuristic algorithm is quite low.

\section{REFERENCES}

[1] S. Song and A. C. Singer, "Pilot-aided OFDM channel estimation in the presence of the guard band," IEEE Trans. Commun., vol. 55, no. 8, pp. 1459-1465, Aug. 2007.

[2] L. Hanzo, M. Münster, B. J. Choi, and T. Keller, OFDM and MC-CDMA for Broadband Multi-User Communications, WLANs and Broadcasting. New York, NY, USA: Wiley, 2003.

[3] M. Morelli and U. Mengali, "A comparison of pilot-aided channel estimation methods for OFDM systems," IEEE Trans. Signal Process., vol. 49, no. 12, pp. 3065-3073, Dec. 2001.

[4] J.-W. Choi and Y.-H. Lee, "Optimal pilot pattern for channel estimation in OFDM systems," IEEE Trans. Wireless Commun., vol. 4, no. 5, pp. 2083-2088, Sep. 2005.

[5] H. Tang, K. Y. Lau, and R. W. Brodersen, "Interpolation-based maximum likelihood channel estimation using OFDM pilot symbols," in Proc. IEEE GLOBECOM 2002, pp. 1860-1864.

[6] O. Simeone, Y. Bar-Ness, and U. Spagnolini, "Pilot-based channel estimation for OFDM systems by tracking the delay-subspace," IEEE Trans. Wireless Comm., vol. 3, no. 1, pp. 315-325, Jan. 2004.

[7] X. D. Dong, W.-S. Lu, and A. C. K. Soong, "Linear interpolation in pilot symbol assisted channel estimation for OFDM," IEEE Trans. Wireless Comm., vol. 6, no. 5, pp. 1910-1920, May 2007.

[8] R. Nehi and J. M. Cioff, "Pilot tone selection for channel estimation in a mobile OFDM system," IEEE Trans. Consum. Electron., vol. 44, no. 3, pp. 1122-1128, Aug. 1998.

[9] M. Dong, L. Tong, and B. M. Sadler, "Optimal pilot placement for channel tracking in OFDM," in Proc. MILCOM 2002, pp. 602-606.

[10] X. Cai and G. B. Giannakis, "Error probability minimizing pilots for OFDM with M-PSK modulation over Rayleigh-fading channels," IEEE Trans. Veh. Technol., vol. 53, no. 1, pp. 146-155, Jan. 2004. 
[11] P. Pakrooh, A. Amini, and F. Marvasti, "OFDM pilot allocation for sparse channel estimation," in EURASIP J. Adv. Signal Process., 2012, vol. 59, 10.1186/1687-6180-2012-59.

[12] M. D. Larsen, G. Seco-Granados, and A. L. Swindlehurst, "Pilot optimization for time-delay and channel estimation in OFDM systems," in Proc. IEEE Int. Conf. Acoust., Speech, Signal Process. (ICASSP), pp. 3564-3567.

[13] R. Baxley, J. Kleider, and G. T. Zhou, "Pilot design for OFDM with null edge subcarriers," IEEE Trans. Wireless Commun., vol. 8, no. 1, pp. 396-405, Jan. 2009.

[14] S. Ohno, E. Manasseh, and M. Nakamoto, "Preamble and pilot symbol design for channel estimation in OFDM systems with null subcarriers," in EURASIP J. Wireless Commun. Netw., 2011, vol. 2, 10.1186/16871499-2011-2.

[15] B. Muquet and Z. Wang et al., "Cyclic prefixing or zero padding for wireless multicarrier transmissions?," IEEE Trans. Commun., vol. 50 , no. 12, pp. 2136-2148, Dec. 2002.

[16] H. Steendam and M. Moeneclaey, "Different guard interval techniques for OFDM: Performance comparison," in Proc. MC-SS 2007, pp. 11-24.

[17] J. Song, Z. Yang, L. Yang, K. Gong, C. Pan, J. Wang, and Y. Wu, "Technical review on chinese digital terrestrial television broadcasting standard and measurements on some working modes," IEEE Trans. Broadcast., vol. 53, no. 1, p. 17, Mar. 2007.

[18] H. Steendam, "How to select the pilot carrier positions in CP-OFDM?," in Proc. ICC 2013, pp. 1741-1746.

[19] A. H. Land and A. G. Doig, "An Automatic Method of Solving Discrete Programming Problems," Econometrica, vol. 28, no. 3, pp. 497-520, 1960, 10.2307/1910129.

[20] T. Weise, Global Optimization Algorithms-Theory and Application 2009 [Online]. Available: http://www.it-weise.de/, e-book.
[21] H. L. Van Trees, Detection, Estimation and Modulation Theory. New York, NY, USA: Wiley, 1968.

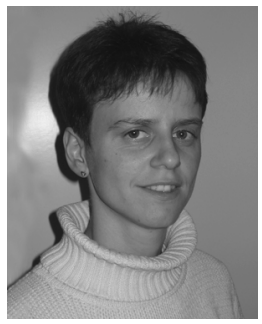

Heidi Steendam (M'01-SM'06) received the M.Sc. degree in Electrical Engineering and the Ph.D. degree in Applied Sciences from Ghent University, Ghent, Belgium in 1995 and 2000, respectively. Since September 1995, she has been with the Digital Communications (DIGCOM) Research Group, Department of Telecommunications and Information Processing (TELIN), Faculty of Engineering, Ghent University, Belgium, first in the framework of various research projects, and since October 2002 as a full time Professor in the area of Digital Communications.

Her main research interests are in statistical communication theory, carrier and symbol synchronization, bandwidth-efficient modulation and coding, spread-spectrum (multi-carrier spread-spectrum), satellite and mobile communication, cognitive radio and cooperative networks. She is the author of more than 125 scientific papers in international journals and conference proceedings.

Since 2002, she is an executive Committee Member of the IEEE Communications and Vehicular Technology Society Joint Chapter, Benelux Section, and since 2012 the vice chair. She has been active in various international conferences as Technical Program Committee chair/member and Session chair. In 2004 and 2011, she was the conference chair of the IEEE Symposium on Communications and Vehicular Technology in the Benelux. She is associate editor of IEEE TRANSACTIONS ON COMMUNICATIONS, EURASIP Journal on Wireless Communications and Networking, and Journal on Communications and Network. 